\title{
Intraabdominal follicular dendritic cell sarcoma: a
}

\section{case report}

\begin{abstract}
Neoplasms of follicular dendritic cells (FDCS) are uncommon and majority of them occur in the lymph nodes. Rarely may they occur inside the abdominal cavity. An intraabdominal location is associated with particularly worse clinical course. The differential diagnoses includes several malignant stromal tumors. A 30 years old male presented with an intrabdominal mass gradually increasing in size since 6 months. $\mathrm{He}$ underwent a CT scan, following which mass was partially removed and sent in multiple pieces for histopathological examination. The histopathological diagnosis was malignant spindle cell tumor and further evaluation of tumor by immunohistochemical studies was advised. The immunohistochemical markers established the diagnosis of Follicular dendritic cell sarcoma. This case report is an attempt to describe histological characteristics of follicular dendritic cell sarcoma and discuss probable differential diagnosis
\end{abstract}

Keywords: follicular dendritic cell sarcoma, intraabdominal, immunohistochemistry

\author{
Volume 2 Issue 4 - 2017
}

\section{Koirala S, Prasad KBR}

Department of Pathology, Nepal Medical College Teaching Hospital, Nepal

Correspondence: Koirala S, Department of Pathology, Nepal Medical College Teaching Hospital, Nepal,

Email sudhamsu.koirala@gmail.com

Received: June 23, 2017 | Published: September 19, 2017

\section{Introduction}

Kojima et al. \& Chen et al. ${ }^{1}$ in the year 1982 jointly gave the name Follicular dendritic cells to the non lymphoid cells in germinal centers with desmonemes. ${ }^{1}$ Tew et al. ${ }^{2}$ defined the functional significance of these cells in that they trap and retain the antigen antibody complexes on their surface and express Fc and $\mathrm{C} 3$ receptors. ${ }^{2}$ So far numerous research into origin of Follicular dendritic cells have failed to decipher the origin of FDCs. ${ }^{3}$ The existence of a primary neoplasm of the follicular dendritic cell was first recognized in 1986 by Monda et al. ${ }^{4}$ FDCS is grouped with the histiocytic and dendritic cell neoplasm by the World Health Organization classification of tumor. ${ }^{5}$ Approximately 150 cases have been reported in English literature so far. ${ }^{6}$ There is a wide age range with mean age of presentation being 44 years. ${ }^{5}$ The most common presentation is lymph node enlargement (64\%), but the frequency of extra nodal occurrence is probably underestimated because on assessment of morphology and site of presentation they are likely to be misdiagnosed as other sarcomas. Chan JK et al. ${ }^{7}$ in his assessment of 17 cases of FDCS considered it to be at least of intermediate grade malignancy. ${ }^{7}$ The exact aetiology of FDC tumour is unknown. A minority of nodal cases developed with pre-existing or simultaneous Castleman's disease of hyaline-vascular type. ${ }^{7}$ Complete excision, if feasible, is the recommended treatment for FDC sarcomas. Intraabdominal tumors, because of their documented aggressive behavior, require adjuvant chemotherapy.

\section{Case report}

A 30 years old male presented to surgical Outpatient department with history of loss of weight, abdominal pain and intraabdominal lump that increased in size over a period of 6months. There was no history of vomiting. On examination of the abdomen, there was a mass occupying the epigastric and left hypochondrium regions which was firm in consistency. On clinical examination, mass was $15 \mathrm{X} 12 \mathrm{~cm}$. On CT scan mass measured $18 \mathrm{X} 13 \mathrm{X} 11 \mathrm{~cm}$. It was predominantly solid with few loculated cystic areas. It was seen displacing the surrounding viscera and showed arterial phase enhancement after administration of contrast (Figure 1). Perigastric and mesenteric lymphadenopathy were also noted. The radiological differential diagnoses of mass were
Intraperitonealdesmoid and mesenteric soft tissue sarcoma. Initially Laparatomy and complete excision of tumor was attempted, however, as complete excision was not possible debulking and adjuvant chemotherapy was considered a better option. Following the surgery, mass was sent in multiple pieces largest measuring $8 \times 5.5 \times 2 \mathrm{~cm}$. Cut section of the mass showed grey white homogeneous areas. Microscopic examination of the mass showed monomorphic spindle cells arranged in multiple storiform areas Figure 2A, short fascicles, whorls and occasional herring bone pattern. These cells showed moderate degree of pleomorphism having oval to spindle nuclei with vesicular chromatin and small but prominent nucleoli. Cytoplasm was moderate to abundant in amount with indistinct cytoplasmic borders. Clustering of nuclei giving an appearance of syncytial pattern was noted. About 10mitosis/10 HPF were noted Figure 2B. There were occasional binucleated and multinucleated cells. Several foci showed infiltration of inflammatory cells namely lymphocytes, neutrophils and occasional plasma cells Figure 2C. Foci of coagulative necrosis were also seen Figure 2D. The report was signed out as malignant spindle cell tumor with advice of immunohistochemistry for exact typing.

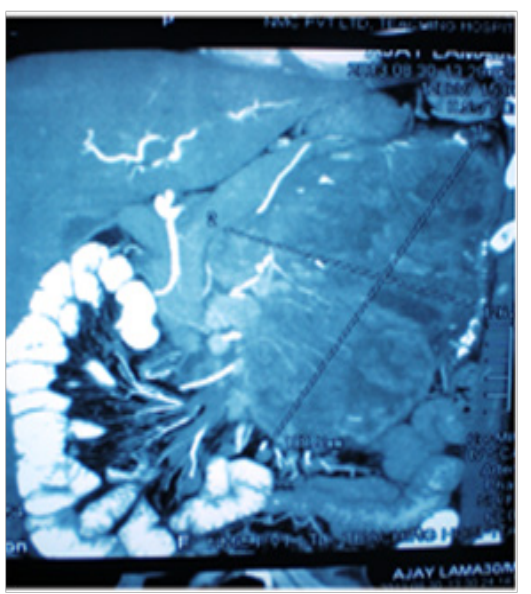

Figure I Tumor mass on coronal section of CT scan. Tumor is seen arising from gut mesentery. 

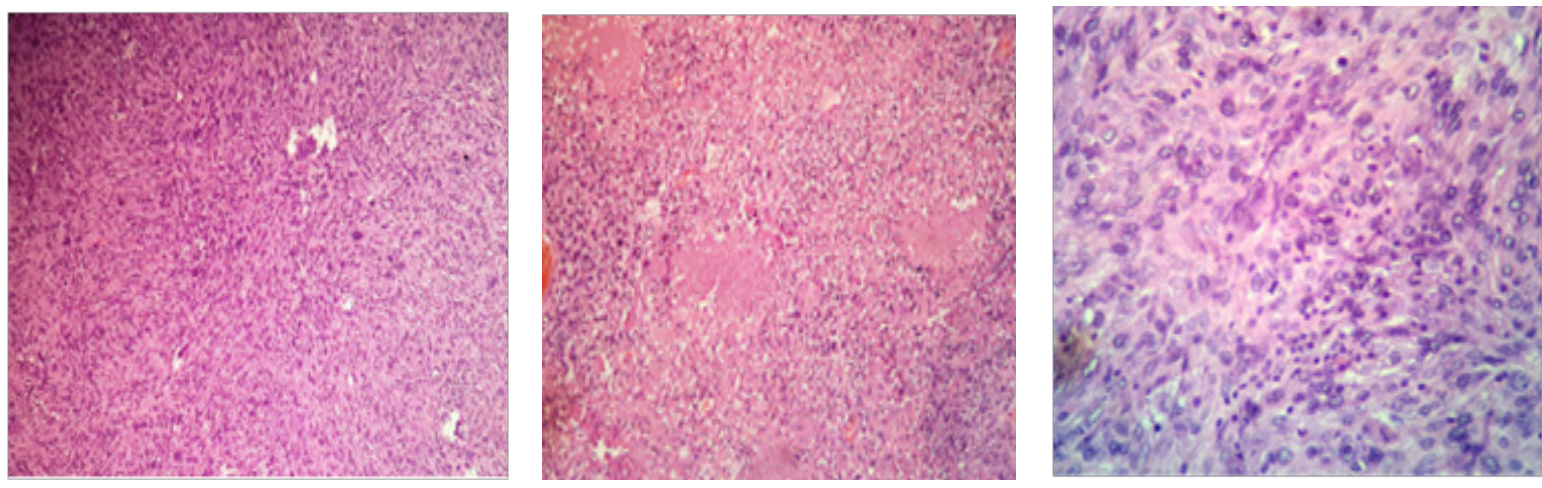

Figure 2 (A) Tumor showing storiform pattern and short fascicles in heamotoxylin eosin stain $X 40$ ).

(B) Low power view showing various foci of necrosis within the tumor in haemotxylin-Eosin (X40).

(C) High power view showing mitosis in the centre with infiltration of tumor by lymphocytes in Haemotxylin-Eosin (X 400).

\section{Discussion}

Recognition of follicular dendritic cell sarcoma requires high degree of suspicion especially when presenting at an extra nodal site. On review of literature presence of spindle cells arranged in storiform pattern, whorls and syncytial pattern with infiltration of tumor by lymphocytes often with perivascular cuffing should raise a suspicion of diagnosis. CD21 and CD35, which recognize complement receptors $\mathrm{C} 3 \mathrm{~d}$ and $\mathrm{C} 3 \mathrm{~b}$, respectively confirm the diagnosis. Ultra structurally, long, complex and occasionally interdigitating cytoplasmic processes joined by desmosomes characterize follicular dendritic cells however ultra structural evidence is seldom required. ${ }^{3}$ Most case reports describe clinical course of FDCS to be similar to low grade malignant tumor. ${ }^{8}$ However Chan JK et al. ${ }^{7}$ in his report describes it to have significant recurrent and metastatic potential thus labeled its nature similar to intermediate grade malignancy. ${ }^{7}$ Some features that are associated with poor outcomes are size of tumor, intraabdominal location, cellular atypia, increasing number of mitosis and coagulative necrosis. ${ }^{7}$ Our case had moderate pleomorphism, 10 mitosis/10 HPF and coagulative necrosis, so probably has poor prognosis Figures 2C \& 2D. Our case was signed as a malignant spindle cell tumor with advice of immunohistochemistry for exact typing of tumor. It is not uncommon that Follicular dendritic cell tumor to be signed out as stromal sarcoma. Chang $\mathrm{KC}$ et al. ${ }^{10}$ described a similar case in which Follicular dendritic cell tumor presenting in colon was diagnosed as stromal tumor with suspicion of gastrointestinal stromal tumor as diagnosis..$^{10}$ The differential diagnosis we had in mind was malignant fibrous histiocytoma with the frequent storiform pattern, bi and multinucleated cells and lymphocytes. However as Chan JK et al. ${ }^{7}$ suggested the severe degree of cellular atypia was lacking. ${ }^{7}$
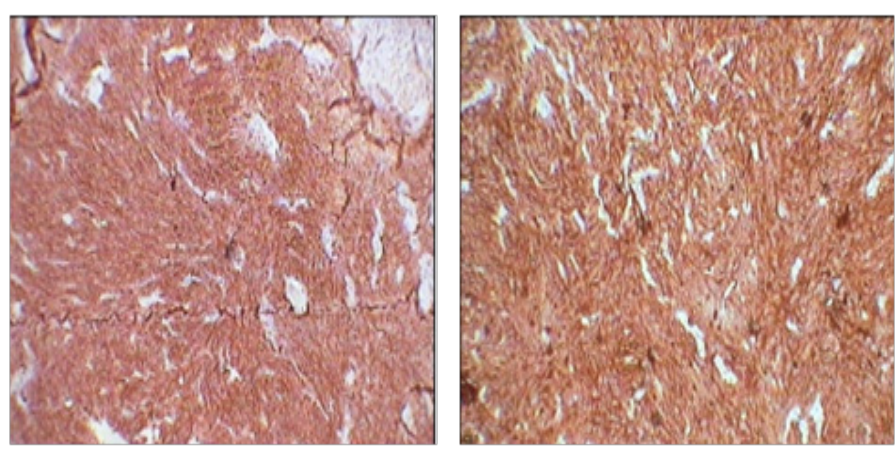

An Intraabodimal location prompted us to believe gastrointestinal stromal tumor as a probable differential diagnosis given the fascicles but gastrointestinal stromal tumor lacks the frequent storiform pattern that were seen in our case. Staining for CD-117 was negative. High power evaluation of tumor showed syncytial cells with vesicular nuclei small but prominent nucleoli mixed with lymphocytes giving an appearance of lymphoepithelioma as differential diagnosis (Figure 2C) Lymphoepithelioma like areas also occur in Follicular dendritic cells sarcoma as mentioned by Chan $\mathrm{JK}$ et al. ${ }^{7}$ As there are large numbers of possible differential diagnosis detail immunohistological examination to type the tumor is necessary. Most of the reports show $100 \%$ reactivity for $\mathrm{CD} 21$ and $\mathrm{CD} 35$. In an international review article composed by multiple authors CD68 and S-100 was present in half of the cases reviewed. CD45 and EMA were detected in few cases. The sprinkled lymphocytes are B-cells, T-cells or mixture of both. The Ki67 index ranged from $1 \%$ to $25 \%$ (average 13\%). ${ }^{11}$

Our case was positive for CD21 Figure 3A, CD23 Figure 3B, S-100 positive Figure 3C and focal EMA positive Figure 3D, Ki67 index was $10-12 \%$. Tumor was negative for desmin, smooth muscle actin, CD-34 and CD-117. These results were similar to reviews on immunohistochemiocal findings mentioned in literature. Choice of treat ment given in literature are total excision of tumor in cases presenting as nodal enlargement and surgery followed by chemotherapy and radiation if the disease is extranodal. ${ }^{5}$ Our case underwent debulking of tumor and is currently the patient is in his third cycle of chemotherapy. Prognostic studies on tumor are limited as a consequence of relatively rare diagnosis. Intra abdominal tumor however is associated with a poor prognosis. In one case of FDCS presenting in head and neck region there was no evidence of disease after 10 years of follow up. ${ }^{12}$
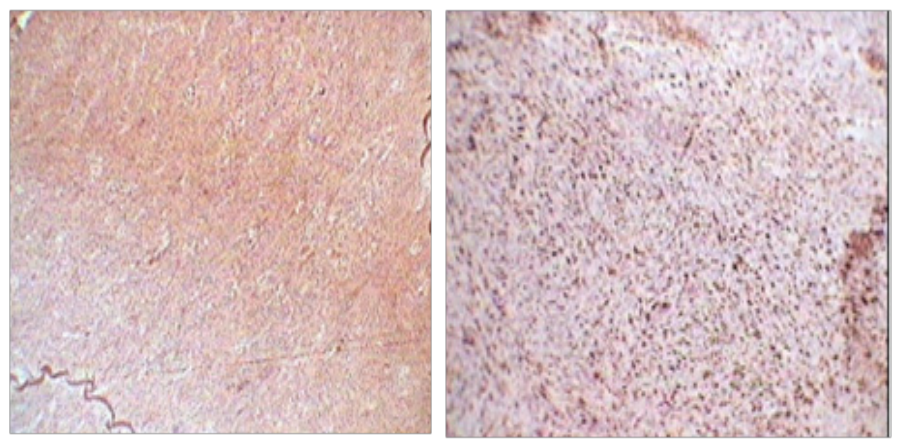

Figure 3 (A) CD 21 positivity (XI0). (B) CD 23 positivity (XI0). (C) Focal EMA positive (XI0). (D) S- 100 positive (XI0). 


\section{Conclusion}

Follicular dendritic cell sarcoma should enter a differential diagnosis of any tumor with frequent storiform pattern, whorls and syncytial arrangement. Infiltration by sprinkled lymphocytes and perivascular cuffing by these lymphocytes should further raise the suspicion of FDCS, regardless of the site. However given its rare occurrence, in comparison to other tumors presenting with similar patterns, the diagnosis can only be confirmed by judicious use of immunohistochemical stains.

\section{Acknowledgements}

None.

\section{Conflict of interest}

The author declares no conflict of interest.

\section{References}

1. Chen LL, Adams JC, Steinman RM. Anatomy of germinal centers in mouse spleen, with special reference to 'follicular dendritic cells. J Cell Biol. 1978;77(1):148-164.

2. Tew JG, Thorbecke J, Steinman RM. Dendritic cells in the immune response: A report from the reticuloendothelial soci- ety committee on nomenclature. J Reticuloendothel Soc committee. 1982;31(5):371-380.

3. Lmai Y, Yamakawa M. Morphology, function and pathology of follicular dendritic cells. Pathol Int. 1996;46(11):807-833.

4. Monda L, Warnke R, Rosai J. A primary lymph node malignancy with features suggestive of dendritic reticulum cell differentiation: a report of 4 cases. Am J Pathol. 1986;122(3):562-572.
5. Chan JKC, Pileri SA, Delsoi G, et al. World Health Organization Classification of Tumours of Hematopoietic and lymphoid tissues. In: Swerdlow S, Elias C editor. Lyon, France: IARC press; 2008. 363 p.

6. Chan JKC, Tsang WYW, Ng CS. Follicular dendritic cell tumor and vascular neoplasm complicating hyaline-vascular disease. Am J Surg Pathol. 1994;18(5):517-525.

7. Chan J KC, Fletcher CDM, Nayler SJ, et al. Follicular Dendritic Cell Sarcoma: clinicopathologic analysis of 17 cases suggesting a malignant potential higher than currently recognized. Cancer. 1997;79(2):294313.

8. Wang H, Su Z, Hub Z, et al. Follicular dendritic cell sarcoma: a report of six cases and a review of the chinese literature. Diagn pathol. 2010;5:67.

9. JIan W, Yunyi K, Hongfen L, et al. Two cases of follicular dendritic cell sarcoma. Chinese medical Journal. 2003;116(5):794-797.

10. Chang KC, Jin YT, Chen FF, et al. Follicular dendritic cell sarcoma of the colon mimicking stromal tumour. Histopathology. 2001;38(1):2529.

11. Pileri SA, Grogan TM, Harris NL, et al. Tumours of histiocytes and accessory dendritic cells: an immunohistochemical approach to classification from the international lymphoma study group based on 61 cases. Histopathology. 2002;41:1-29.

12. Chera BS, Orlando C, Villaret DB, et al. Follicular dendritic cell sarcoma of the head and neck: case report and literature review. Laryngoscope. 2008;118(9):1607-1612. 